\title{
Association between routine and standardized blood pressure measurements and left ventricular hypertrophy among patients on hemodialysis
}

\author{
Jaspreet Khangura', Bruce F Culleton², Braden J Manns'2,3, Jianguo Zhang2', Lianne Barnieh33, Michael Walsh33, \\ Scott W Klarenbach ${ }^{4}$, Marcello Tonelli, ${ }^{4}$, Magdalena Sarna², Brenda R Hemmelgarn*2,3 for the Alberta Kidney Disease \\ Network
}

\begin{abstract}
Background: Left ventricular (LV) hypertrophy is common among patients on hemodialysis. While a relationship between blood pressure (BP) and LV hypertrophy has been established, it is unclear which BP measurement method is the strongest correlate of LV hypertrophy. We sought to determine agreement between various blood pressure measurement methods, as well as identify which method was the strongest correlate of LV hypertrophy among patients on hemodialysis.

Methods: This was a post-hoc analysis of data from a randomized controlled trial. We evaluated the agreement between seven BP measurement methods: standardized measurement at baseline; single pre- and post-dialysis, as well as mean intra-dialytic measurement at baseline; and cumulative pre-, intra- and post-dialysis readings (an average of 12 monthly readings based on a single day per month). Agreement was assessed using Lin's concordance correlation coefficient (CCC) and the Bland Altman method. Association between BP measurement method and LV hypertrophy on baseline cardiac MRI was determined using receiver operating characteristic curves and area under the curve (AUC).
\end{abstract}

Results: Agreement between BP measurement methods in the 39 patients on hemodialysis varied considerably, from a CCC of 0.35 to 0.94 , with overlapping $95 \%$ confidence intervals. Pre-dialysis measurements were the weakest predictors of LV hypertrophy while standardized, post- and inter-dialytic measurements had similar and strong (AUC 0.79 to 0.80 ) predictive power for LV hypertrophy.

Conclusions: A single standardized BP has strong predictive power for LV hypertrophy and performs just as well as more resource intensive cumulative measurements, whereas pre-dialysis blood pressure measurements have the weakest predictive power for LV hypertrophy. Current guidelines, which recommend using pre-dialysis measurements, should be revisited to confirm these results.

\section{Background}

Hypertension is common among patients on hemodialysis (HD) and is associated with an increased risk of coronary artery disease, congestive heart failure, cerebrovascular complications, mortality and left-ventricular (LV) hypertrophy[1-3]. Cardiovascular disease accounts for the majority of deaths in patients with end stage renal disease (ESRD)[4,5], thus adequate blood

\footnotetext{
* Correspondence: brenda.hemmelgarn@albertahealthservices.ca

2 Department of Medicine, University of Calgary, Calgary, Canada Full list of author information is available at the end of the article
}

pressure (BP) control is important to reduce the risk of adverse cardiac events. LV hypertrophy, in itself, affects up to $80 \%$ of ESRD patients[2], is an established cardiac manifestation of chronic hypertension[6] and an independent predictor of cardiovascular events and mortality in both the general and ESRD population[7,8]. Although the relationship between hypertension and cardiovascular morbidity and mortality is not consistently reported in the HD patient population[9], it is generally accepted that BP control is important for cardiovascular risk reduction in these patients[10]. Recommendations for BP measure- 
ment among patients on dialysis, including timing (prevs. post-dialysis), target levels, and methods of measurement, are variable, while in general, blood pressure control in HD patients remains poor $[1,11]$.

Although the most accurate BP measurement technique among patients on HD is unknown, it has been demonstrated that casual dialysis-unit BP measurements differ considerably when compared to ambulatory and standardized BP measurements[12-14], and correlate poorly with target organ damage[13]. Further, it was shown that BP measurements obtained outside the dialysis unit (home and ambulatory BP measurements) were more strongly correlated with LV hypertrophy than those obtained within the dialysis unit[12,13]. Previous studies have produced inconsistent results with respect to the BP measurement method which best predicts LV hypertrophy[15,16].

By using more sensitive measures of LV mass, and more frequent measurements of $\mathrm{BP}$, we sought to determine the agreement between various BP measurement methods, at different times (pre-, intra- and post- dialysis) among patients on HD. We also sought to determine the association between BP measurement methods and LV hypertrophy. Further, we hypothesized that BP values obtained by a standardized BP protocol (as recommended by national guidelines) would be more closely associated with LV hypertrophy than BP values obtained by other techniques.

\section{Methods}

\section{Subjects}

This study involves a post-hoc analysis of data collected within a previously reported randomized controlled trial, where details of the subjects and protocol are previously described[17]. Subjects included in this analysis were limited to those from the University of Calgary who underwent cardiac magnetic resonance imaging (cMR) as a baseline procedure during the clinical trial, but prior to the intervention. Subjects from the University of Alberta were excluded because of the absence of serial dialysisassociated BP measurements.

Eligible subjects were 18 years of age or older, receiving in-center, self-care or home hemodialysis three times a week. The study protocol was approved by the University of Calgary bioethics committee.

\section{Standardized BP at baseline}

Standardized BP measurements were obtained at baseline by a physician using a mercury sphygmomanometer in accordance with the Canadian Hypertension Education Programs protocol for blood pressure measurement[18]. Three sitting BP measurements were taken 5 minutes apart, after the patient had been resting in a quiet room for at least 5 minutes. The average of the last two measurements was utilized as the standardized BP value for this study[17]. The standardized BP measurements were obtained prior to dialysis but within two weeks of the cMR exam.

\section{Casual BP measurements at a single hemodialysis session at baseline}

A single pre- and post dialysis BP measurement was taken by a dialysis unit nurse with patients in a sitting position, within 30 minutes prior to and following the dialysis session using either the automated GAMBRO Phoenix or manually using a mercury sphygmometer on the non-fistula arm. Intra-dialytic BPs were recorded every 15-30 minutes during dialysis. The average value of all of the BP measures taken during a single dialysis session, which ranged from 2 to 11 readings per dialysis session (median 7), was used to determine the intra-dialysis BP.

\section{Cumulative BP measurements}

Monthly pre-, intra- and post-dialysis BP measurements were retrospectively collected from hemodialysis records for a single hemodialysis session each month during the 12 month period prior to the cMR examination. These casual BP measurements were obtained by dialysis staff or by the patient (for those patients on home conventional hemodialysis).

\section{Left ventricular mass and left ventricular hypertrophy}

cMR was performed on 1.5-T MRI systems (Avanto or Sonata; Siemens Medical Solutions, Erlangen, Germany) with 8-channel cardiac coils. Standard, breath-held, retrospectively electrocardiogram-gated gradient-echo sequences (steady state free precession, SSFP) in contiguous short-axis views ( 25 phases, slice thickness $8 \mathrm{~mm}$ ) were applied. The evaluation of the cMR images was performed in a professional core lab (CIRCLE International Ltd., Calgary, Canada) by readers blinded to BP measurement data. Papillary muscles were included in the LV myocardial mass calculation. The formula by DuBois and DuBois[19] was used to index LV mass to body surface area. The presence of LV hypertrophy was defined as LV mass $/ \mathrm{m}^{2}>83$ for males and $\mathrm{LV}$ mass $/ \mathrm{m}^{2}>67$ for females[20].

\section{Laboratory measurements}

Lab records for the 12 months prior to the cMR procedure were extracted from the Southern Alberta Renal Program database. Given the small sample size, we chose, a priori, to limit our assessment to serum calcium, phosphate, hemoglobin and parathyroid hormone (PTH) as these have been linked to alterations in LV structure[21]. Serum calcium was not corrected for serum albumin values. PTH was measured using the Nichols bio-intact 
assay. Mean values were collected at baseline and in the 12 months prior to the cMR.

\section{Statistical analysis}

Subject characteristics are presented as mean \pm standard deviation (SD) or median and inter-quartile range (IQR) for continuous variables as appropriate, and number (percent) for categorical data. All BP measurements are reported as mean $\pm \mathrm{SD}$. Agreement between BP measurement methods was assessed using two statistical methods. Lin's[22,23] concordance correlation coefficient (CCC) was used to measure overall agreement between any two measurement methods. Lin's CCC incorporates both bias and precision, with a CCC value of 1 indicating perfect agreement and -1 indicating perfect inverse agreement. CCC values are reported with their respective 95\% confidence intervals (CI). The second statistical method used to assess agreement was the Bland and Altman method[24], which plots the difference between pairs of measurements on the $y$-axis against the mean of each pair on the $\mathrm{x}$-axis. The $95 \% \mathrm{CI}$ are also presented. Pearson's product moment correlation coefficients are reported for each method against LV mass, standardized to body surface area.

Multivariate linear regression analyses, with LV mass corrected for body surface area as the dependent variable and BP parameters as independent variables, were used to determine the potential confounding effects of hemoglobin, calcium-phosphate product, $\mathrm{PTH}$, age and dialysis vintage. Receiver operated characteristic (ROC) curves, and area under the curve (AUC) statistics, with their respective $95 \% \mathrm{CI}$, were generated to compare the test performances of various BP methods in detecting the presence of LV hypertrophy. Only the results for systolic blood pressure (SBP) are shown as the results of the diastolic blood pressure analysis did not appreciably change the outcomes of this study. All analyses were performed using Stata 9.2 (Stata Corporation, College Station, Texas).

\section{Results}

\section{Patient and baseline characteristics}

A total of 39 patients were included in the study. The mean age of study subjects was 54 years and two-thirds were male (Table 1 ). The majority of subjects were taking antihypertensive medications, the most common being angiotensin converting enzyme (ACE) inhibitors or angiotensin receptor blockers. LV hypertrophy, as measured by cMR, existed in 29 (74.4\%) study subjects. Blood pressure results obtained by the various methods are detailed in Figure 1. Pre-dialysis BP values were higher than post-dialysis BP values, and both baseline and cumulative pre-dialysis BPs were higher than the standardized BP taken pre dialysis.

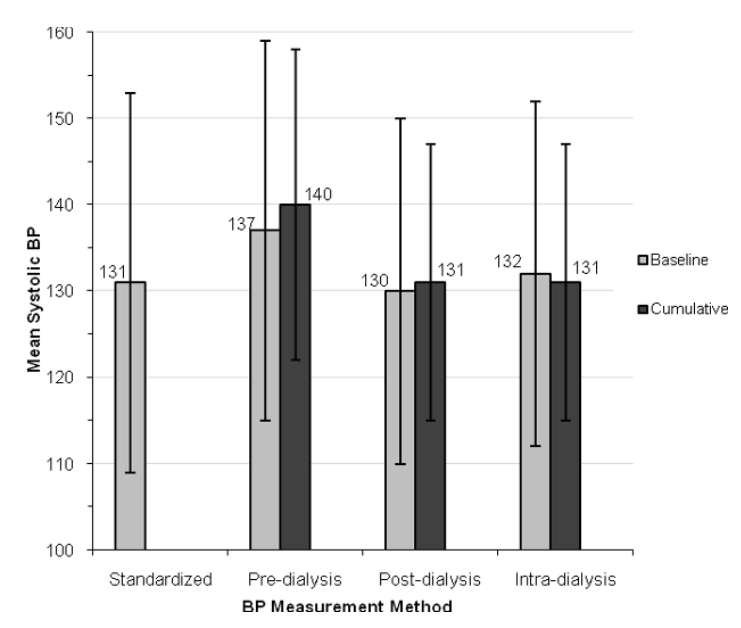

Figure 1 Summary of systolic blood pressure measurements by different methods $(n=39)$

\section{Agreement between BP measurement methods}

The agreement levels for the different methods for SBP measurements are shown in Table 2. Agreement between methods was highly variable, from a CCC of 0.35 (95\% CI $0.05,0.59)$ for baseline pre-dialysis and standardized methods to a CCC of 0.94 ( $(95 \%$ CI $0.89,0.97)$ for cumulative intra-dialysis and cumulative post-dialysis methods. The 95\% CI for the CCC's for all methods overlapped, suggesting no significant difference in agreement between BP measurement methods. The Bland-Altman analysis confirmed this, showing no consistent bias between the different methods.

Although not statistically significant, standardized, post- and intra-dialysis measurements (baseline and cumulative) had moderate to high agreement (CCCs 0.67 to 0.94 ), while the comparison of each method with baseline pre-dialysis BPs showed lower levels of agreement (CCCs 0.35 to 0.62 ).

\section{Association between BP measurement method and LV mass}

Correlations between BP measurement methods and LV mass are shown in Table 3. Standardized BP had a correlation of $\mathrm{r}=0.44(\mathrm{P}=0.005)$. Baseline post- and intradialysis methods had correlations of $0.60(\mathrm{P}=0.0001)$ and $0.59(\mathrm{P}<0.0001)$ respectively, slightly higher than the correlation for cumulative post- and intra- dialysis methods. The correlation between BP method and LV mass was significant $(P<0.05)$ for all methods except baseline predialysis $(r=0.30, P=0.068)$. Results were unchanged when adjusted for hemoglobin, calcium-phosphate product, PTH, age and dialysis vintage.

ROC curves for the SBP measurements and LV hypertrophy are shown in Figure 2 and their respective AUC 
Table 1: Baseline characteristics of study subjects

\begin{tabular}{|c|c|}
\hline Characteristic $^{\dagger}$ & $\mathbf{N}=39$ \\
\hline Age, years & $54.4 \pm 13.3$ \\
\hline Male gender, n (\%) & $26(66.6)$ \\
\hline Caucasian, n (\%) & $33(84.6)$ \\
\hline Body mass index $\left(\mathrm{kg} / \mathrm{m}^{2}\right)$ & $25.3 \pm 5.4$ \\
\hline Time on dialysis, years & $5.0 \pm 4.6$ \\
\hline Prior renal transplantation, $\mathrm{n}(\%)$ & $12(30.8)$ \\
\hline \multicolumn{2}{|l|}{ Cause of ESRD, $n$ (\%) } \\
\hline Diabetic nephropathy & $12(30.0)$ \\
\hline Hypertension/vascular & $3(7.7)$ \\
\hline Glomerulonephritis & $10(25.6)$ \\
\hline Polycystic kidney disease & $4(10.3)$ \\
\hline Urologic & $4(10.3)$ \\
\hline Other & $6(15.4)$ \\
\hline \multicolumn{2}{|l|}{ Comorbid illnesses, n (\%) } \\
\hline Ischemic heart disease & $17(43.6)$ \\
\hline Congestive heart failure & $10(25.6)$ \\
\hline Peripheral vascular disease & $6(15.4)$ \\
\hline Cerebrovascular disease & $6(15.4)$ \\
\hline Diabetes mellitus & $16(41.0)$ \\
\hline \multicolumn{2}{|l|}{ Medication use, $\mathrm{n}(\%)$} \\
\hline ASA & $17(54)$ \\
\hline ACE inhibitor or ATII antagonist & $25(64)$ \\
\hline Calcium channel blocker & $21(54)$ \\
\hline Beta-blocker & $16(41)$ \\
\hline Other anti-hypertensive & $5(13)$ \\
\hline Any anti-hypertensive & $33(85)$ \\
\hline Hemoglobin, $\mathrm{g} / \mathrm{dL}$ & $121 \pm 13.7$ \\
\hline Serum calcium, mmol/L & $2.32 \pm 0.24$ \\
\hline Serum phosphate, $\mathrm{mmol} / \mathrm{L}$ & $1.69 \pm 0.43$ \\
\hline Calcium-phosphate product, $\mathrm{mmol}^{2} /: \mathrm{L}^{2}$ & $3.94 \pm 1.10$ \\
\hline Parathyroid hormone, $\mathrm{pg} / \mathrm{ml}$ & $239(83,391)$ \\
\hline Left ventricular mass & $173.7 \pm 61.0$ \\
\hline Left ventricular mass $/ \mathrm{m}^{2}$ & $93.5 \pm 31.6$ \\
\hline Left ventricular hypertrophy, $\mathrm{n}(\%)$ & $29(74.4 \%)$ \\
\hline
\end{tabular}

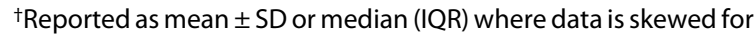
continuous variables and number (\%) for categorical data. values are provided in Table 3. All the SBP values were related to LV hypertrophy. All of the BP measurement methods had similar AUC values and their 95\% CIs overlapped; hence they were not significantly different from each other. AUC values were highest for standardized systolic (AUC 0.79), baseline post- (AUC 0.79) and baseline intra-dialysis (AUC 0.80). Both cumulative intra- and post-dialysis methods had an AUC of 0.74 while the AUC for the baseline pre-dialysis method was 0.71 .

\section{Discussion}

In this study we investigated the agreement between standardized and casual (single dialysis session and 12 month cumulative) BP measurement methods, and their association with left ventricular mass in hemodialysis patients. Current guidelines $[25,26]$ recommend the use of a single pre-dialysis BP measurement for the care and management of chronic hemodialysis patients. However our analysis of pre-, intra-, post- and standardized BP measurements suggests that a single pre-dialysis measurement is the least accurate method of BP assessment and demonstrates the weakest performance at predicting LV hypertrophy compared to cumulative and standardized measurements.

Intra- or post- dialysis BP measurements collected over several months may provide a more accurate assessment of $\mathrm{BP}$ in a stable, chronic HD patient rather than a single pre-dialysis measurement. However, this is resource intensive and often impractical in the clinical setting. Furthermore, in spite of extremely high agreement (CCC of $0.94)$, cumulative intra- and post-dialysis BP measurements were not the strongest predictors of LV hypertrophy, which is the more clinically significant outcome. BP values obtained by the standardized BP measurement protocol were closely associated with LV mass, but no more so than a single intra-dialysis or single post-dialysis measurement, all three of which had similar and strong predictive power for determining LV hypertrophy.

While the relationship between BP and mortality in ESRD patients is not clear[9,27,28], it is well accepted that high BP contributes to LV hypertrophy - which itself is an independent risk factor for morbidity and mortality in the ESRD population[7,8]. The prevalence of LV hypertrophy in our study, $74.4 \%$, is similar to that reported in prior studies[13,29].

Two previous studies of BP as a predictor of LV geometry both found pre-dialysis BP's to be strong determinants of LV hypertrophy[15,16]. These studies however were small, used a less sensitive measure of LV mass (echocardiography), and only measured BP over 12 dialysis sessions. A more recent study of 140 hemodialysis 
Table 2: Agreement levels between different methods for all systolic blood pressure measurements.

\begin{tabular}{|c|c|c|c|c|c|c|c|c|}
\hline & & \multicolumn{4}{|c|}{ Baseline measurements } & \multicolumn{3}{|c|}{ Cumulative measurements } \\
\hline & & Standard & Pre-dialysis & Post-dialysis & Intra-dialysis & Pre-dialysis & Post-dialysis & Intra-dialysis \\
\hline & Standardized & & & & & & & \\
\hline & & $-5.8(-13.9,2.2)$ & & & & & & \\
\hline \multirow[t]{9}{*}{ Baseline } & Pre-dialysis & $(-54.5,42.9)$ & --- & --- & --- & --- & --- & --- \\
\hline & & $0.35(0.05,0.59)$ & & & & & & \\
\hline & & $1.0(-5.3,7.3)$ & $6.8(0.4,13.2)$ & & & & & \\
\hline & Post-dialysis & $(-37.0,39.0)$ & $(-31.9,45.5)$ & --- & --- & --- & --- & --- \\
\hline & & $0.58(0.33,0.75)$ & $0.52(0.27,0.71)$ & & & & & \\
\hline & & $-1.1(-7.0,4.7)$ & $4.6(-1.8,11.0)$ & $-2.2(-5.5,1.0)$ & & & & \\
\hline & Intra-dialysis & $(-37.6,35.1)$ & $(-34.0,43.2)$ & $(-21.8,17.3)$ & --- & --- & --- & --- \\
\hline & & $0.62(0.39,0.78)$ & $0.55(0.29,0.73)$ & $0.87(0.77,0.93)$ & & & & \\
\hline & & $-8.6(-14.3,-2.8)$ & $-2.7(-8.4,2.9)$ & $-9.6(-15.0,-4.1)$ & $-7.3(-12.1,-2.5)$ & & & \\
\hline \multirow[t]{8}{*}{ Cumulative } & Pre-dialysis & $(-43.2,26.1)$ & $(-36.7,31.2)$ & $(-42.5,23.3)$ & $(-36.4,21.8)$ & --- & --- & --- \\
\hline & & $0.57(0.34,0.73)$ & $0.62(0.39,0.78)$ & $0.54(0.31,0.71)$ & $0.65(0.45,0.79)$ & & & \\
\hline & & $0.6(-4.5,5.8)$ & $6.5(0.2,12.8)$ & $-0.36(-5.1,4.4)$ & $1.9(-2.6,6,3)$ & $9.2(5.5,12.9)$ & & \\
\hline & Post-dialysis & $(-30.4,31.7)$ & $(-31.6044 .5)$ & $(-29.1,28.4)$ & $(-25.1,28.8)$ & $(-13.2,31.6)$ & --- & --- \\
\hline & & $0.67(0.47,0.80)$ & $0.46(0.20,0.66)$ & $0.67(0.47,0.81)$ & $0.72(0.54,0.84)$ & $0.67(0.49,0.80)$ & & \\
\hline & & $0.2(-5.0,5.4)$ & $6.0(0.0,12.0)$ & $-0.8(-5.4,3.8)$ & $1.4(-2.4,5.3)$ & $8.8(5.8,11.7)$ & $-0.4(-2.3,1.3)$ & \\
\hline & Intra-dialysis & $(-31.2,31.6)$ & $(-30.4,42.4)$ & $(-28.5,27.0)$ & $(-21.9,24.8)$ & $(-9.1,26.6)$ & $(-11.4,10.5)$ & --- \\
\hline & & $0.65(0.46,0.79)$ & $0.49(0.25,0.68)$ & $0.69(0.50,0.82)$ & $0.78(0.64,0.87)$ & $0.75(0.61,0.85)$ & $0.94(0.89,0.97)$ & \\
\hline
\end{tabular}

Each cell contains the mean difference ( $95 \%$ confidence interval) (first row), $95 \%$ agreement limits (second row) and CCC (95\% confidence interval) (third row).

${ }^{\dagger}$ Mean difference was calculated by subtracting the row from the column.

patients reported that out-of-clinic recordings (1-weekaveraged home BP readings) performed better at predicting LV hypertrophy than routine and standardized BP recordings obtained pre- and post-dialysis in the dialysis unit[13]. Unfortunately, access to home BP monitors or supervised ambulatory BP monitoring is not always available and convincing patients to measure BP at home when it is measured so frequently in HD units, may be difficult.

BP management in HD patients is partly dependent on the ability of physicians to accurately assess BP levels, which remains a challenge. In the absence of a universally accepted method for BP assessment among patients on hemodialysis, we selected standardized BP as the gold standard for the purpose of this study, as it was based on a protocol established and endorsed by national hypertension guidelines[18]. Previous studies provide conflicting results as to whether pre-dialysis, post-dialysis, interdialytic or a combination of BP measurements methods are most valuable for clinical decision making[15,30,31]. However the issue of accuracy of BP measurement in the dialysis unit is often ignored. Our results are consistent with previous reports $[11-14,32]$ suggesting that routine dialysis BP measurements are in fact highly inaccurate.

Our results should be interpreted in the context of the study limitations. Firstly, our sample size was small, including 39 patients. Despite the small sample size, our use of cMR enabled us to use a more accurate measure of LV mass; thus our point estimates are more precise than those of previous studies[13]. Secondly, as a single-centre 
Table 3: Pearson's product correlation coefficients for baseline and cumulative systolic BP measurement methods with $\mathrm{LVM} / \mathrm{m}^{2+}$ and ROC statistics for LV hypertrophy ${ }^{\dagger+}$

\begin{tabular}{|c|c|c|c|c|}
\hline \multicolumn{2}{|c|}{ BP Measurement Method } & \multirow{2}{*}{\multicolumn{2}{|c|}{$\begin{array}{c}\text { Correlation with LVM/m } 2 \\
\text { Pearson's r P value }\end{array}$}} & \multirow{3}{*}{$\begin{array}{l}\text { ROC AUC (95\% Confidence Interval) } \\
0.79(0.64,0.94)\end{array}$} \\
\hline & & & & \\
\hline & Standardized & 0.44 & 0.005 & \\
\hline \multirow{3}{*}{ Baseline } & Pre-dialysis & 0.30 & 0.068 & $0.71(0.53,0.89$ \\
\hline & Post-dialysis & 0.60 & 0.0001 & $0.79(0.64,0.93)$ \\
\hline & Intra-dialysis & 0.59 & $<0.0001$ & $0.80(0.64,0.96)$ \\
\hline \multirow{3}{*}{ Cumulative } & Pre-dialysis & 0.38 & $<0.0001$ & $0.78(0.62,0.95)$ \\
\hline & Post-dialysis & 0.53 & $<0.0001$ & $0.74(0.57,0.92)$ \\
\hline & Intra-dialysis & 0.51 & 0.0009 & $0.74(0.58,0.90)$ \\
\hline
\end{tabular}

$+\mathrm{LVM} / \mathrm{m}^{2}=\mathrm{LVM}$ indexed to patient's BSA, using the formula described by DuBois and DuBois ${ }^{19}$

${ }^{+\dagger} \mathrm{LV}$ hypertrophy was defined as an LVM/m ${ }^{2}>83$ for males and $\mathrm{LVM} / \mathrm{m}^{2}>67$ for females ${ }^{20}$

observational study, the generalizability of our results could be questioned. However the lenient inclusion and exclusion criteria does increase generalizability of the results. The cross-sectional nature of the data for baseline BP measurement, lack of ambulatory blood pressure measurements and assessment of LV hypertrophy without an assessment of volume status prevents us from drawing firm conclusions, however, the results are further supported by cumulative BP measurements obtained over a 12 month period. Finally, our study included
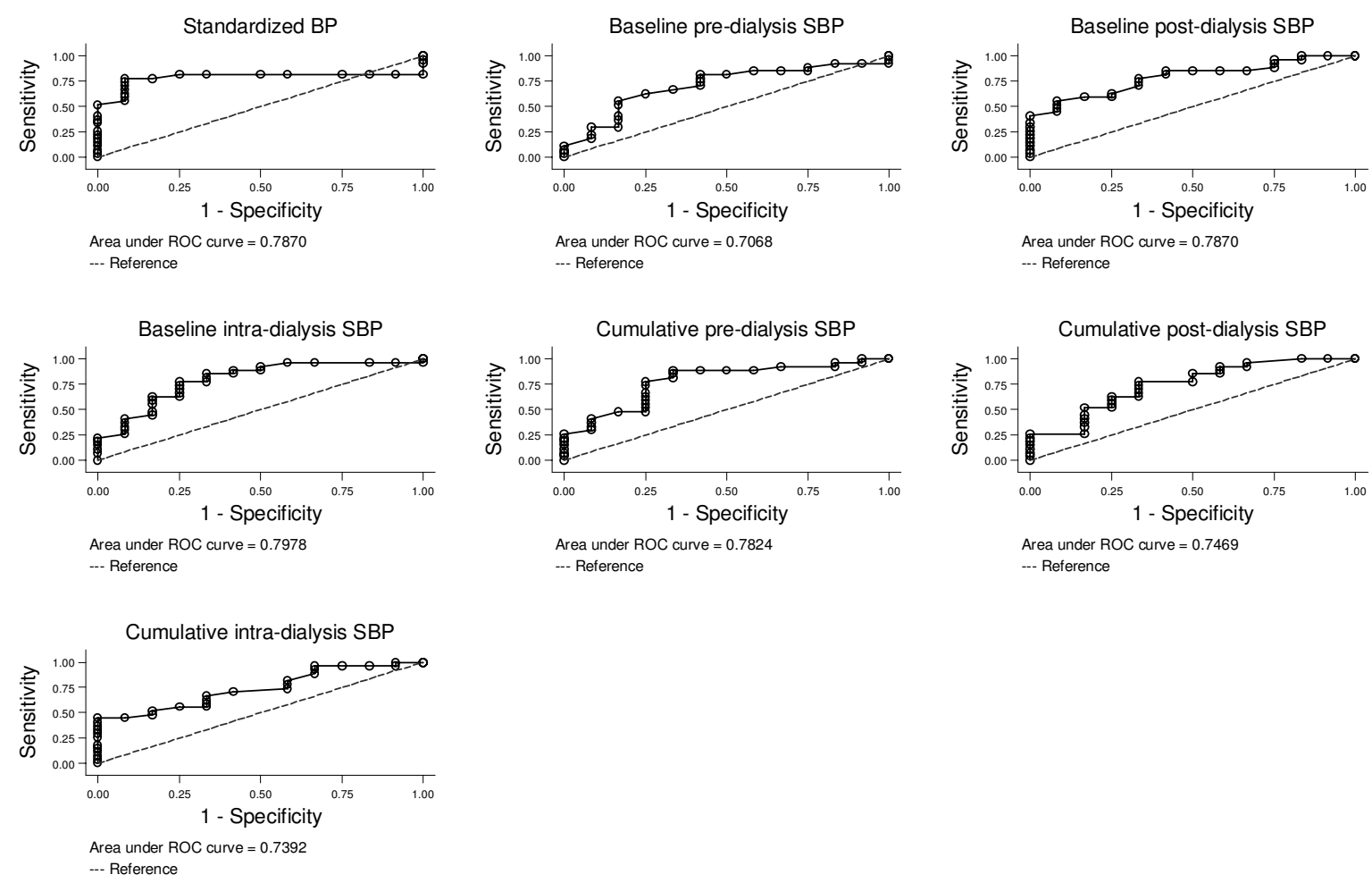

Area under ROC

Area under ROC curve $=0.7469$ - Reference

Figure 2 ROC curves for different systolic BP measurement methods. The diagonal dotted line indicates a hypothetical test with no predictive value 
patients with a wide range of ESRD etiologies, including diabetes and heart failure, both of which contribute to LV hypertrophy. The autonomic and cardiovascular dysfunction in these disease processes could have confounded the BP-LV hypertrophy correlation. Further studies are necessary to delineate whether these co-morbidities affect the BP-LV hypertrophy relationship.

\section{Conclusion}

In summary, we found that a single standardized BP measurement has strong predictive power for LV hypertrophy, but is not significantly better than casual post- and intra-dialysis BP measurements taken at a single point in time or over several months. For ease of practice, a single standardized measurement performs just as well as the more resource intensive cumulative measurements. Given the poor agreement with other methods, and relatively poor predictive power of a single pre-dialysis measurement in predicting LV hypertrophy, the results of this study also suggest that the current practice guidelines, which advocate the use of pre-dialysis measurements for chronic hemodialysis patients, warrants further study. This study does not however provide strong evidence to disregard current guidelines.

\begin{abstract}
Abbreviations
HD: hemodialysis; LV: left ventricular; ESRD: end stage renal disease; BP: blood pressure; CMR: cardiac MRI; PTH: parathyroid hormone; SD: standard deviation IQR: inter-quartile range; CCC: concordance correlation coefficient; ROC: receiver operated characteristic; AUC: area under the curve; $\mathrm{Cl}$ : confidence intervals; SBP: systolic blood pressure; ACE: angiotensin converting enzyme
\end{abstract}

\section{Competing interests}

The authors declare that they have no competing interests.

\section{Authors' contributions}

$\mathrm{BRH}, \mathrm{MT}, \mathrm{SWK}, \mathrm{BJM}$, and BFC made substantial contributions to conception, design and acquisition of data and funds. JP, BRH, JZ, BJM and BFC made substantial contributions to conception and design. All authors made substantial contributions to interpretation of the data, all authors were involved in revising the manuscript for important intellectual content, and all authors have read and approved the final manuscript.

\section{Acknowledgements}

This study was funded by a grant from the Kidney Foundation of Canada. JK was supported by a Summer Studentship Award from the Canadian Hypertension Society. BJM, MT and BRH were supported by New Investigator awards from the Canadian Institutes of Health Research. MT, SWK and BRH were supported by Population Health Investigator awards from the Alberta Heritage Foundation for Medical Research. MWW was supported by the Kidney Research Scientist Core Education Training Program and an Alberta Heritage Foundation for Medical Research Award. At the time this work was done, BFC was an Associate Professor at the University of Calgary. He is now with Baxter Corporation, McGaw Park, Illinois. LB is supported by an Allied Health Fellowship Award from the Kidney Foundation of Canada.

\section{Author Details}

'Department of Medicine, University of British Columbia, Vancouver, Canada, 2Department of Medicine, University of Calgary, Calgary, Canada, ${ }^{3}$ Department of Community Health Sciences, University of Calgary, Calgary, Canada and ${ }^{4}$ Department of Medicine, University of Alberta, Edmonton, Canada

Received: 15 March 2010 Accepted: 24 June 2010

Published: 24 June 2010

\section{References}

1. Agarwal R, Nissenson A, Battle D, Coyne D, Trout J, Warnock D: Prevalence, treatment, and control of hypertension in chronic hemodialysis patients in the United States. Am J Med 2003, 115:291-297.

2. Horhl M, Horhl W: Hemodialysis-associated hypertension: Pathophysiology and therapy. Am J of Kid Dis 2002, 39:227-244.

3. Foley R, Parfrey P: Cardiovascular disease and mortality in ESRD. Nephrol 1998, 11:239-245.

4. US Renal Data System: USRDS 1997 Annual Data Report. In Book USRDS 1997 Annual Data Report City: The National Institutes of Health, National Institute of Diabetes and Digestive and Kidney Diseases; 1997

5. Canadian Organ Renal Replacement Register: Canadian Institute for Health Information Annual Report 1997. In Book Canadian Institute for Health Information Annual Report 1997 Volume 1. City: Canadian Institute for Health Information; 1997.

6. Dahlof B: Left ventricular hypertrophy and angiotensin II antagonists. Am J Hypertens 2001, 14:174-182.

7. Silberberg J, Barre P, Prichard S, Sniderman A: Impact of left ventricular hypertrophy on survival in end-stage renal disease. Kidney Int 1989, 36:286-290

8. Foley R, Parfrey P, Harnett J, Kent G, Murray D, Barre P: The prognostic importance of left ventricular geometry in uremic cardiomyopathy. Am Soc Nephrol 1995, 5:2024-2031

9. Stidley C, Hunt W, Tentori F, Schmidt D, Rohrscheib M, Paine S, Bedrick E, Meyer $\mathrm{K}$, Johnson $\mathrm{H}$, Zager $\mathrm{P}$ : Changing relationship of blood pressure with mortality over time among hemodialysis patients. J Am SoC Nephrol 2006, 17:513-520.

10. Agarwal R: Assessment of blood pressure in hemodialysis patients. Semin Dial 2002, 15:299-304

11. Rahman M, Dixit A, Donley V, Gupta S, Hanslik T, Lacson E, Ogunidepe A Weigel K, Smith M: Factors associated with inadequate blood pressure control in hypertensive hemodialysis patients. Am J Kidney Dis 1999, 33:498-506.

12. Agarwal R, Andersen M, Bishu K, Saha C: Home blood pressure monitoring improves the diagnosis of hypertension in hemodialysis patients. Kidney Int 2006, 69:900-906.

13. Agarwal R, Brim N, Mahenthiran J, Andersen M, Saha C: Out-ofhemodialysis-unit blood pressure is a superior determinant of left ventricular hypertrophy. Hypertension 2006, 47:62-68.

14. Rahman M, Griffin V, Kumar A, Manzoor F, Wright JJ, Smith M: A comparison of standardized versus 'usual' blood pressure measurements in hemodialysis patients. Am J Kidney Dis 2002 39:1226-1230

15. Conlon P, Walshe J, Heinle S, Minda S, Krucoff M, Schwab S: Predialysis systolic blood pressure correlates with mean 24 hour systolic blood pressure and left ventricular mass in stable hemodialysis patients. $J$ Am Soc Nephrol 1996, 7:2658-2663

16. Zoccali C, Mallamaci F, Tripepi G, Benedetto F, Cottini E, Giacone G, Malatino L: Prediction of left ventricular geometry by clinic, pre-dialysis and 24-h ambulatory blood pressure monitoring in hemodialysis patients. Journal of Hypertension 1999, 17:1751-1758.

17. Culleton B, Walsh M, Klarenbach S, Mortis G, Scott-Douglas N, Quinn R, Tonelli M, Donnelly S, Friedrich M, Kumar A, et al.: Effect of frequent nocturnal hemodialysis vs conventional hemodialysis on left ventricular mass and quality of life: A randomized controlled trial. JAMA 2007, 298:1291-1299.

18. Hemmelgarn B, McAllister F, Myers M, McKay D, Bolli P, Abbott C, Schiffrin E, Grover S, Honos G, Lebel M, et al:: The 2005 Canadian Hypertension Education Program recommendations for the management of hypertension: Part 1 - Blood pressure measurement, diagnosis and assessment of risk. Can J Cardiol 2005, 21:645-656.

19. DuBois D, DuBois E: A formula to estimate the approximate surface area if height and weight be known. Arch Intern Med 1916, 17:863-871.

20. Alfakih K, Plein S, Thiele H, Jones T, Ridgway J, Sivananthan M: Normal human left and right ventricular dimensions for MRI as assessed by turbo gradient echo and steady-state free precession imaging sequences. J Magn Reson Imaging 2003, 17:323-329.

21. Parfrey P, Foley R, Harnett J, Kent G, Murray D, Barre P: Outcome and risk factors for left ventricular disorders in chronic uremia. Nephrol Dial Transplant 1996, 11:1277-1285. 
22. Lin L: A concordance correlation coefficient to evaluate reproducibility. Biometrics 1989, 45:255-268.

23. Lin L: A note on the concordance correlation coefficient. Biometrics 2000, 56:324-325

24. Bland J, Altman D: Statistical methods for assessing agreement between two methods of clinical measurement. Lancet 1986, 1:307-310.

25. Clinical practice guidelines for cardiovascular disease in dialysis patients Section II guideines on management of cardiovascular risk factors Guideline 12: BP [http://www.kidney.org/professionals/kdogi/ guidelines $\mathrm{cvd} /$ quide $12 \mathrm{htm}$

26. Jindal K, Chan C, Dzeiel C, Hirsch D, Soroka S, Tonelli M, Culleton B: Hemodialysis clinical practice guidelines for the Canadian Society of Nephrology. J Am Soc Nephrol 2006, 17:S1-S27.

27. Foley R, Herzog C, Collins A: Blood pressure and long-term mortality in United States hemodialysis patients: USRDS waves 3 and 4 study. Kidney Int 2002, 62:1784-1790.

28. Klassen P, Lowrie E, Reddan D, DeLong E, Coladonato J, Szczech L, Lazarus J, Owen WJ: Association between pulse pressure and mortality in patients undergoing maintenance hemodialysis. JAMA 2002, 287:1548-1555.

29. Foley R, Parfrey P, Harnett J, Kent G, Martin C, Murray D, Barre P: Clinical and echocardiographic disease in patients starting endstage renal disease therapy. Kidney Int 1995, 47:186-192.

30. Mailloux L, Haley W: Hypertension in the ESRD patient. Am J Kidney Dis 1998, 32:705-719.

31. Kooman J, Gladziwa U, Bocker G, Winjen J, Bortel L, Luik A, de Leeuw P, van Hoff J, Leunissen $\mathrm{K}$ : Blood pressure during the interdialytic period in hemodialysis patients: estimation of representative blood pressure values. Nephrol Dial Transplant 1992, 7:917-923.

32. Agarwal R, Lewis R: Prediction of hypertension in chronic hemodialysis patients. Kidney Int 2001, 60:1982-1989.

\section{Pre-publication history}

The pre-publication history for this paper can be accessed here: http://www.biomedcentral.com/1471-2369/11/13/prepub

doi: 10.1186/1471-2369-11-13

Cite this article as: Khangura et al., Association between routine and standardized blood pressure measurements and left ventricular hypertrophy among patients on hemodialysis BMC Nephrology 2010, 11:13

Submit your next manuscript to BioMed Centra and take full advantage of:

- Convenient online submission

- Thorough peer review

- No space constraints or color figure charges

- Immediate publication on acceptance

- Inclusion in PubMed, CAS, Scopus and Google Scholar

- Research which is freely available for redistribution

Submit your manuscript at www.biomedcentral.com/submit
C Biomed Central 